\title{
Morcegos (Chiroptera) capturados em lagoas do Parque Nacional da Restinga de Jurubatiba, Rio de Janeiro, Brasil
}

\author{
Júlia Lins Luz ${ }^{1,3}$, Renato Mangolin ${ }^{2}$, Carlos Eduardo Lustosa Esbérard ${ }^{1}$ \& Helena de Godoy Bergallo ${ }^{2}$ \\ ${ }^{1}$ Laboratório de Diversidade de Morcegos, Instituto de Biologia, \\ Universidade Federal Rural do Estado do Rio de Janeiro - UFRRJ, \\ Km 47 da antiga estrada Rio-São Paulo, CP 74507, CEP 23890-000, Seropédica, RJ, Brasil \\ ${ }^{2}$ Departamento de Ecologia, Instituto de Biologia, Universidade do Estado do Rio de Janeiro - UERJ, \\ Rua São Francisco Xavier, 524, CEP 20559-900, Rio de Janeiro, RJ, Brasil \\ ${ }^{3}$ Autor para correspondência: Júlia Lins Luz,e-mail: julialinsluz@yahoo.com.br
}

LUZ, J.L., MANGOLIN, R., ESBÉRARD, C.E.L. \& BERGALLO, H.G. Bats (Chiroptera) sampled in lagoons at Parque Nacional da Restinga Jurubatiba, Rio de Janeiro, Brazil. Biota Neotrop. 11(4): http://www. biotaneotropica.org.br/v11n4/en/abstract?inventory+bn01011042011.

\begin{abstract}
The aim of this study was to perform a list of bat species captured in six lagoons in the Parque Nacional da Restinga de Jurubatiba, Rio de Janeiro State, Brazil. The park is located about $180 \mathrm{~km}$ eastward of Rio de Janeiro city and covers the municipalities of Macaé, Carapebus and Quissamã. The region is dominated by sand dunes and sand ridges formed in the Quaternary. We captured 149 bats belonging to three families and 14 species. Most species caught in restinga de Jurubatiba were frugivores, partly due to technique employed, mist-net, that favors the capture of individuals of the Phyllostomidae family. Five species are uncommonly captured in bats inventories (Uroderma magnirostrum, Lasiurus cinereus, Lophostoma brasiliense, Chiroderma villosum and Diaemus youngi). Species caught varied among lagoons, showing the importance of these habitats for maintaining the diversity of bats.

Keywords: Atlantic forest, lagoon, richness, guild, assemblage, Phyllostomidae.
\end{abstract}

LUZ, J.L., MANGOLIN, R., ESBÉRARD, C.E.L. \& BERGALLO, H.G. Morcegos (Chiroptera) capturados em lagoas do Parque Nacional da Restinga de Jurubatiba, Rio de Janeiro, Brasil. Biota Neotrop. 11(4): http://www.biotaneotropica.org.br/v11n4/pt/abstract?inventory+bn01011042011.

Resumo: O objetivo deste trabalho foi elaborar uma lista das espécies de quirópteros capturados em seis lagoas no Parque Nacional da Restinga de Jurubatiba, Estado do Rio de Janeiro, Brasil. O Parque está localizado a cerca de $180 \mathrm{~km}$ a leste da cidade do Rio de Janeiro e abrange os municípios de Macaé, Carapebus e Quissamã. A região é dominada por dunas e cordões arenosos formados no Quaternário. Foram capturados 149 morcegos, pertencentes a três famílias e 14 espécies. A maior parte das espécies capturadas na restinga de Jurubatiba foi de frugívoros, em parte devido à técnica empregada, com redes de neblina, que favorece a captura de indivíduos da família Phyllostomidae. Foram capturadas cinco espécies pouco frequentes em inventários de morcegos (Uroderma magnirostrum, Lasiurus cinereus, Lophostoma brasiliense, Chiroderma villosum e Diaemus youngi). As espécies capturadas variaram entre as lagoas, o que mostra a importância desses ambientes para a manutenção da diversidade de morcegos.

Palavras-chaves: Mata Atlântica, lagoa, riqueza, guilda, taxocenose, Phyllostomidae. 


\section{Introdução}

Os ecossistemas costeiros que compõem as restingas têm sido submetidos a um intenso processo de degradação (Lacerda \& Esteves 2000, Tanizaki-Fonseca \& Moulton 2000) e são ambientes frágeis em razão da natureza de seu solo caracteristicamente pobre, composto principalmente por areia não consolidada e, em muitas áreas, com considerável grau de salinidade (Hay et al. 1981). A região costeira é umas das áreas mais alteradas e exploradas do país (Rocha et al. 2004). Atualmente, está cada vez mais restrita a ocorrência de extensas áreas contínuas de restinga ao longo da costa do Brasil, uma vez que elas se encontram muito fragmentadas (Lacerda \& Esteves 2000, Rocha et al. 2004). Apesar da ameaça real à existência desses ecossistemas, somente nas últimas duas décadas as restingas brasileiras têm sido alvo de estudos mais sistematizados, com levantamentos florísticos e faunísticos (e.g., Cerqueira 2000, Lacerda \& Esteves 2000, Pereira \& Araújo 2000, Rocha 2000, Bergallo et al. 2004). Poucas áreas exclusivamente de restingas são protegidas em Unidades de Conservação (UCs), como o Parque Estadual de Setiba (ES) e o Parque Nacional da Restinga de Jurubatiba (RJ).

Estudos sobre quirópteros nas restingas brasileiras são escassos e tiveram início na década de 1990, com o trabalho de Cerqueira et al. (1990) na Restinga da Barra de Maricá, RJ. Nesse trabalho, Cerqueira et al. (1990) capturaram um único indivíduo da espécie Glossophaga soricina (Pallas, 1766) e ainda, observaram o forrageamento da espécie Noctilio leporinus (Linnaeus, 1758) sobre uma coleção de água. No entanto, alguns estudos recentes têm mostrado o crescente interesse por parte dos pesquisadores nesses ecossistemas. Luz et al. (2009) publicaram uma lista de 17 espécies de morcegos para a restinga de Praia de Neves, no Estado do Espírito Santo e Carvalho et al. (2009) uma lista com nove espécies para uma área de restinga no município de Jaguaruna, Santa Catarina. O esforço amostral nestes estudos e, consequentemente, o número de indivíduos capturados foram bastante reduzidos. Oprea et al. (2009) e Nogueira et al. (2010) reuniram informações disponíveis sobre inventários de morcegos de restinga e verificaram que o esforço amostral realizado neste ambiente ainda é ínfimo se comparado a levantamentos realizados em áreas florestadas da Mata Atlântica.

O Parque Nacional da Restinga de Jurubatiba representa a maior área de restinga do Estado do Rio de Janeiro e é o primeiro Parque Nacional no Brasil a compreender exclusivamente o ecossistema de restinga. Jurubatiba é a área de restinga mais bem preservada do país e encontra-se praticamente intacta. No Estado do Rio de Janeiro, poucas áreas de restingas encontram-se em Unidades de Conservação sendo esse parque de extrema importância para a preservação desse ecossistema (Rocha et al. 2004).

Até 2009 apenas sete espécies de morcegos eram conhecidas para o Parque Nacional da Restinga de Jurubatiba (Bergallo et al. 2004, Mangolin et al. 2007, Costa et al. 2008). Esses indivíduos também fazem parte do presente estudo sendo no total 23 capturas: Artibeus lituratus (Olfers, 1818) $(\mathrm{n}=12)$, Lophostoma brasiliense Peters, 1867 ( $\mathrm{n}=3)$, Carollia perspicillata (Linnaeus, 1758) $(\mathrm{n}=2)$, Platyrrhinus recifinus (Thomas, 1901) $(\mathrm{n}=2)$, G. soricina $(\mathrm{n}=2)$, Chiroderma villosum Peters, $1860(\mathrm{n}=1)$ e Diaemus youngi (Jentink, 1893) $(\mathrm{n}=1)$. Todas estas espécies, pertencentes à família Phyllostomidae, foram capturadas próximas a Lagoa de Cabiúnas. As capturas dos três indivíduos de Lophostoma brasiliense, em uma única noite, aumentaram a distribuição geográfica desta espécie em cerca de $350 \mathrm{~km}$ para o sul (Mangolin et al. 2007). Atualmente são conhecidas 16 espécies para o parque (Pessôa et al. 2010).

O total de 14 lagoas localiza-se dentro da área do Parque (EnrichPrast et al. 2004). Tais lagoas fazem parte do conjunto denominado por Sofiatti (1998) como lagoas da planície de restinga. Em geral, as lagoas costeiras atuam como filtros que retêm material orgânico e inorgânico oriundos do continente (Enrich-Prast et al. 2004). A salinidade das lagoas encontradas no Parque Nacional da Restinga de Jurubatiba pode variar desde $0,1 \%$ (doce) a 99,5\% (hipersalinas) (Enrich-Prast et al. 2004). As alterações de fatores abióticos, como a salinidade, $\mathrm{pH}$ e temperatura, podem modificar a comunidade biótica existente na lagoa (Caumette 1992). Lagoas com baixa salinidade ou com baixa profundidade, por exemplo, são caracterizadas pela grande deposição de macrofitas aquáticas.

Este estudo tem como objetivo apresentar a lista de espécies capturadas no Parque Nacional da Restinga de Jurubatiba, próximo a seis diferentes lagoas.

\section{Material e Métodos}

O Parque Nacional da Restinga de Jurubatiba (PNRJ), localizado na Região do Petróleo e Gás (sensu Saraça et al. 2007) no Estado do Rio de Janeiro (22 $23^{\circ} \mathrm{S}$ e $\left.41^{\circ} 45^{\prime} \mathrm{O}\right)$, possui área de 14.860 ha e localiza-se nos Municípios de Macaé, Quissamã e Carapebús. O PNRJ destaca-se por possuir em sua área 14 lagoas e 10 comunidades vegetais (Araújo et al. 1998). As lagoas Cabiúnas, Comprida, Amarraboi, Garças, Visgueiro e Piripiri foram selecionadas para a coleta de morcegos por ainda apresentarem um cordão de mata em seu entorno. O mapa da área foi elaborado utilizando a base cartográfica do Instituto Brasileiro de Geografia e Estatística de 1:10.000, através do programa ArcGis 9.0® (Figura 1).

As seis lagoas amostradas são descritas abaixo, de acordo com Caramaschi et al. (2004), Enrich-Prast et al. (2004) e Farjalla et al. (2001):

a) Lagoa Cabiúnas: localizada no Município de Macaé, recebe um constante aporte fluvial. Seu contorno é dendrítico, o que possibilita grandes extensões de vegetação marginal. Pode ter contatos esporádicos com o mar por rompimento eventual da barreira arenosa que os separa, por ressacas ou por percolação;

b) Lagoa Comprida: localizada no Município de Macaé, apresenta a menor área entre as lagoas do parque, com $0,13 \mathrm{~km}^{2}$. Apresenta $\mathrm{pH}$ ácido (ca. 5,54) decorrentes dos compostos húmicos provenientes da drenagem da região, e baixos índices de salinidade. As espécies vegetais dominantes na área são Typha domingensis (Typhaceae), Utricularia foliosa (Lentibulariaceae), Salvinia auriculata (Salviniaceae), Nymphoides humboldtiana (Menyanthaceae) e Nymphaea ampla (Nymphaeaceae);

c) Lagoa Amarra-boi: localizada no Município de Quissamã, é a lagoa com um dos menores graus de salinidade e possui o $\mathrm{pH}$ mais ácido (ca. 3,91). Está suscetível a estiagem na época de seca;

d) Lagoa das Garças: localizada no Município de Quissamã, insere-se na restinga paralelamente ao mar. A distância dessa lagoa ao oceano é a maior dentre as lagoas estudadas. Localiza-se em uma região de restinga entre dunas, com poucas áreas planas, o que permite a formação de poças resistentes à época de estiagem, mantidas provavelmente por lençol freático;

e) Lagoa Piripiri: localizada no Município de Quissamã, também é paralela à costa, porém mais próxima ao mar que a lagoa das Garças. Possui grande suscetibilidade à época de estiagem;

f) Lagoa Visgueiro: localizada no Município de Quissamã, foi formada nas depressões entre as faixas de areia que constitui a restinga. É a lagoa com a maior concentração salina $(71,4 \%)$ e maior $\mathrm{pH}(9,55)$ encontrado no Parque.

As sessões de captura foram realizadas entre os meses de abril e outubro de 2003, incluindo uma noite de coleta piloto em 2001 (total de 12 noites). Minutos antes do crepúsculo as redes de neblina foram abertas nas áreas selecionadas, permanecendo abertas por um período de 6 horas a cada noite. Para a captura de morcegos 


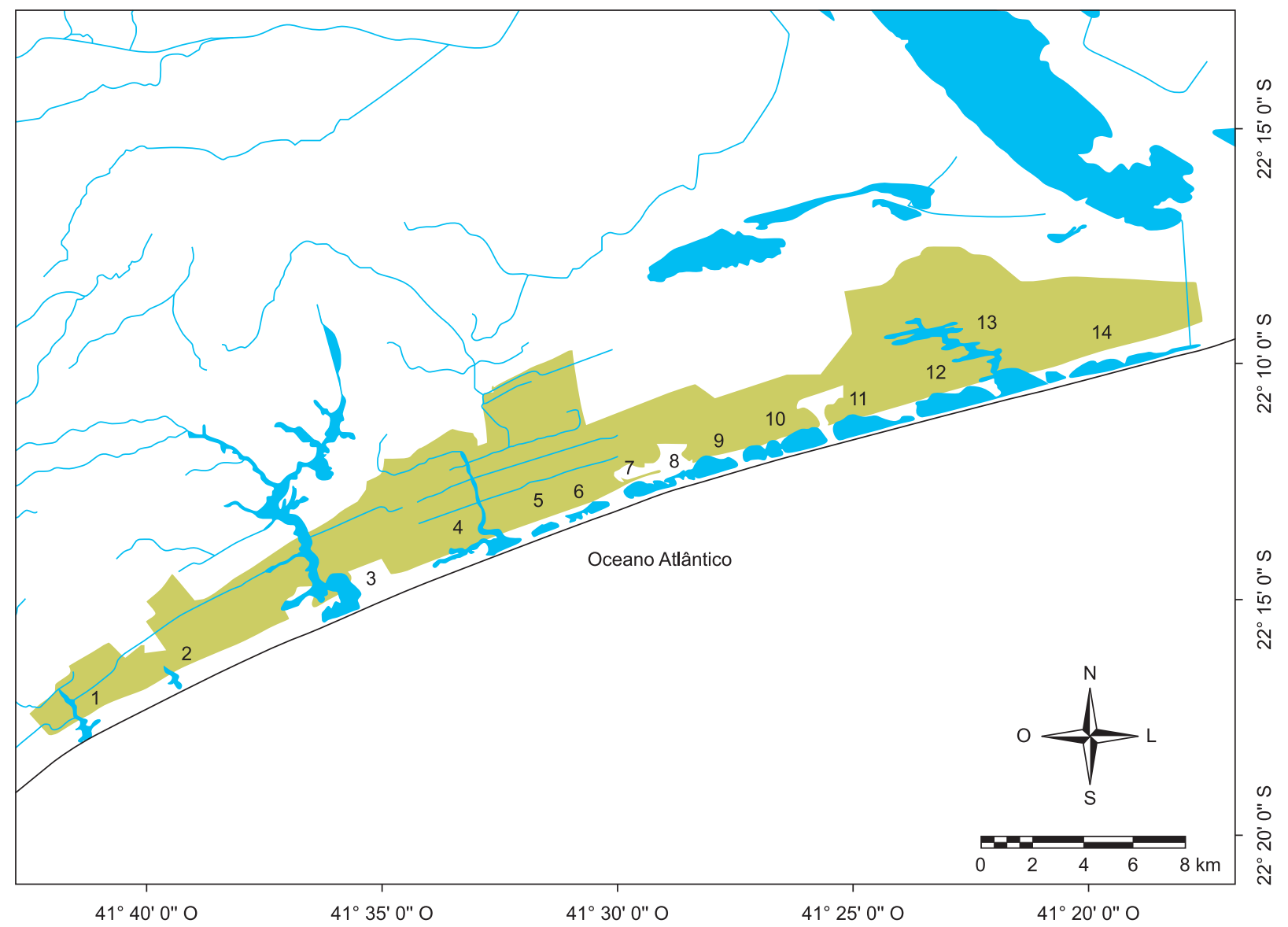

Figura 1. Localização das 14 lagoas encontradas no Parque Nacional da Restinga de Jurubatiba, Estado do Rio de Janeiro, sudeste do Brasil. Legenda das lagoas: 1-Cabiúnas, 2-Comprida, 3-Carapebus, 4-Paulista, 5-Amarra-Boi, 6-Garças, 7-Piripiri II, 8-Piripiri I, 9-Maria-Menina, 10-Robalo, 11-Visgueiro, 12-Pires, 13-Preta e 14-Ubatuba. O estudo foi realizado nas lagoas de número 1, 2, 5, 6, 7 e 11.

Figure 1. Schematic map of the 14 lagoons found in the Parque Nacional da Restinga de Jurubatiba, Rio de Janeiro State, Southeastern Brazil. Legend of the lagoons: 1-Cabiúnas, 2-Comprida , 3-Carapebus, 4-Paulista 5-Amarra-Boi, 6-Garças, 7-Piripiri II, 8-Piripiri I, 9-Maria-Menina, 10-Robalo, 11-Visgueiro, 12-Pires, 13-Preta and 14-Ubatuba. The study was conducted in the lagoons numbers 1, 2, 5, 6, 7 and 11.

utilizamos nove redes, com malha de $36 \mathrm{~mm}$, medindo sete e $12 \mathrm{~m}$ de comprimento e $3 \mathrm{~m}$ de altura, armadas em distintos lugares. Estas foram armadas diretamente sobre a lâmina d'água das lagoas, em regiões brejosas, no entorno das matas associadas às lagoas ou no interior das mesmas matas. As redes foram distribuídas de forma que o maior número de redes armadas permanecesse fora das matas. O esforço de coleta, calculado segundo Straube \& Bianconi (2002), variou de 1.545 a 1.620 h.m $\mathrm{m}^{2}$ por noite, totalizando 19.140 h.m². As redes foram inspecionadas a intervalos médios de 20 minutos. Os animais retirados nas redes foram acondicionados em sacos de algodão durante todo o período da coleta, sendo soltos após o fim das atividades, por volta das 24:00 horas, eliminando a possibilidade de recaptura na mesma noite. Alguns exemplares foram mortos, fixados em formol $10 \% \mathrm{e}$ preservados em álcool 70\%. Esses foram depositados como material testemunho na coleção de referência do Laboratório de Diversidade de Morcegos (Processo 1755/89-IBAMA/SUPES/RJ) do Instituto de Biologia, Universidade Federal Rural do Rio de Janeiro (Apêndice 1).

Visando tentar entender a estruturação da comunidade de morcegos nesse ecossistema de restinga, os morcegos foram classificados em guildas alimentares propostas por Kalko et al. (1996). A abundância das espécies amostradas foi comparada entre as lagoas através do Coeficiente de dissimilaridade de Bray-Curtis e a composição (presença ou ausência) através do Índice de Similaridade de Jaccard. As análises foram feitas no programa Systat 11. Foram feitas duas curvas de acumulação de espécies, uma relacionando a riqueza acumulada com lagoas e outra com número de capturas.

\section{Resultados}

Com uma eficiência de captura de 0,008 capturas $/$ h.m², foram capturados 149 morcegos, pertencentes a três famílias e 14 espécies (Tabela 1). Carollia perspicillata (Linnaeus, 1758) foi a espécie mais frequente, com o maior número de capturas $(36,91 \%)$, seguida de Myotis nigricans (Schinz, 1821) (18,12\%) e Artibeus lituratus (Olfers, 1818) (16,78\%). As espécies Uroderma magnirostrum Davis, 1968, Chiroderma villosum Peters, 1860, Diaemus youngi (Jentink, 1893) e Lasiurus cinereus (Palisot de Beauvois, 1796) foram consideradas espécies localmente raras, com apenas um indivíduo capturado.

Das 14 espécies capturadas, oito têm hábito predominantemente frugívoro. Essa guilda foi a que obteve maior número de capturas e com maior número de espécies, seguida da guilda dos insetívoros aéreos representada por duas espécies. As demais guildas (catadores, nectarívoros, piscívoros e hematófagos) apresentaram apenas uma espécie (Tabela 2). 
Tabela 1. Espécies, número de indivíduos capturados e riqueza de morcegos de seis lagoas amostradas do Parque Nacional da Restinga de Jurubatiba em 2003, Estado do Rio de Janeiro, sudeste do Brasil.

Table 1. Species, number of captured individuals, and bat richness of six lagoons sampled at Parque Nacional da Restinga de Jurubatiba in 2003, Rio de Janeiro State, Southeastern Brazil.

\begin{tabular}{|c|c|c|c|c|c|c|c|}
\hline Espécies & Cabiúna & Garça & Comprida & Visgueiro & Amarra-boi & Piripiri & Total \\
\hline \multicolumn{8}{|l|}{ Família Noctilionidae } \\
\hline Noctilio leporinus (Linnaeus, 1758) & - & - & - & - & 6 & - & 6 \\
\hline \multicolumn{8}{|l|}{ Família Phyllostomidae } \\
\hline Lophostoma brasiliense Peters, 1867 & - & - & - & - & 3 & - & 3 \\
\hline Glossophaga soricina (Pallas, 1766) & 2 & 5 & - & - & 3 & 7 & 17 \\
\hline Carollia perspicillata (Linnaeus, 1758) & 2 & 16 & 17 & 3 & 3 & 14 & 55 \\
\hline Uroderma magnirostrum Davis, 1968 & - & - & 1 & - & - & - & 1 \\
\hline Artibeus lituratus (Olfers, 1818) & 12 & 3 & 1 & - & 1 & 8 & 25 \\
\hline Artibeus fimbriatus Gray, 1838 & - & - & 2 & - & - & 1 & 3 \\
\hline Artibeus obscurus (Schinz, 1821) & - & 1 & 1 & - & - & 1 & 3 \\
\hline Platyrrhinus lineatus (É. Geoffroy, 1810) & - & 1 & - & - & - & 3 & 4 \\
\hline Platyrrhinus recifinus (Thomas, 1901) & 2 & - & - & - & - & - & 2 \\
\hline Chiroderma villosum Peters, 1860 & 1 & - & - & - & - & - & 1 \\
\hline Diaemus youngi (Jentink, 1893) & 1 & - & - & - & - & - & 1 \\
\hline \multicolumn{8}{|l|}{ Família Vespertilionidae } \\
\hline Lasiurus cinereus (Palisot de Beauvois, 1796) & - & - & 1 & - & - & - & 1 \\
\hline Myotis nigricans (Schinz, 1821) & - & 2 & - & 2 & 11 & 12 & 27 \\
\hline Riqueza & 6 & 6 & 6 & 2 & 6 & 7 & 14 \\
\hline
\end{tabular}

Tabela 2. Guildas, abundância, abundância relativa (\%), riqueza de espécies de morcegos e percentual da riqueza local, presentes no Parque Nacional da Restinga de Jurubatiba em 2003, Estado do Rio de Janeiro, sudeste do Brasil.

Table 2. Guilds, Abundance, relative abundance (\%), richness of bats species and percent of local richness, present at the Parque Nacional da Restinga de Jurubatiba in 2003, Rio de Janeiro State, Southeastern Brazil.

\begin{tabular}{lcccc}
\hline \multicolumn{1}{c}{ Guilda } & Capturas & $\begin{array}{c}\text { \% de } \\
\text { capturas }\end{array}$ & Espécies & $\begin{array}{c}\text { \% da } \\
\text { riqueza }\end{array}$ \\
\hline Frugívoros & 94 & 63,09 & 8 & 57,15 \\
Insetívoros & 31 & 20,80 & 3 & 21,43 \\
Nectarívoros & 17 & 11,41 & 1 & 7,14 \\
Piscívoros & 6 & 4,03 & 1 & 7,14 \\
Hematófagos & 1 & 0,67 & 1 & 7,14 \\
\hline
\end{tabular}

A moda e a mediana do número de espécies capturadas por lagoa foi de seis (Tabela 1). As lagoas com a menor (2) e a maior (7) riqueza foram Visgueiro e Piripiri, respectivamente. As lagoas Garças e Piripiri foram as mais similares, tanto na composição (Índice de Jaccard) quanto na abundância das espécies (Coeficiente de BrayCurtis) (Tabela 3). Por outro lado, as lagoas Visgueiro e Comprida foram as mais dissimilares quanto à abundância e composição de espécies (Tabela 3). Em média, as lagoas compartilharam apenas $34,8 \pm 17,6 \%$ das espécies. A lagoa Visgueiro obteve os menores valores em relação a todas as outras lagoas.

Nenhuma das duas curvas de acumulação de espécies, relacionando a riqueza acumulada com lagoas e com número de capturas, apresentou estabilização (Figura 2a,b).

\section{Discussão}

O número de espécies registradas neste estudo foi comparável a outros trabalhos desenvolvidos em restingas. Oprea et al. (2009) registraram 554 capturas de 14 espécies no Parque Estadual Paulo
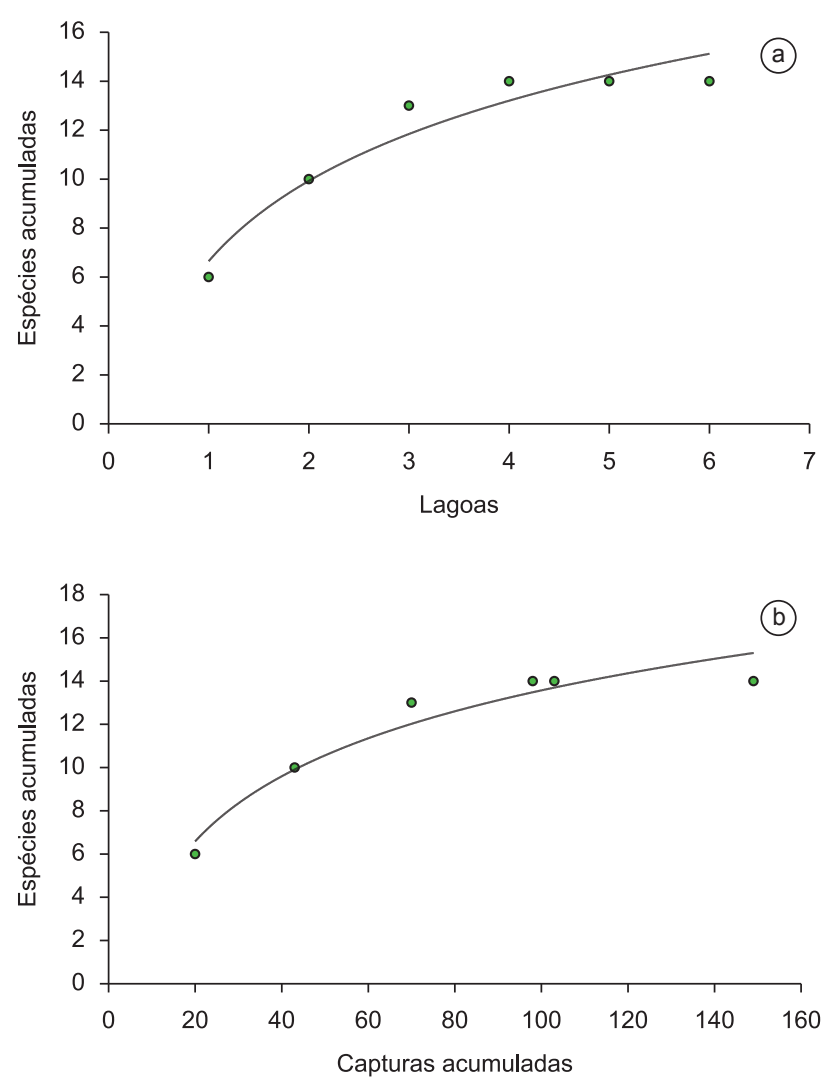

Figura 2. Curva de acumulação de espécies de morcegos em seis lagoas estudadas no Parque Nacional da Restinga de Jurubatiba em 2003, Estado do Rio de Janeiro, sudeste do Brasil: a) riqueza acumulada por lagoas; e b) riqueza acumulada com número de capturas.

Figure 2. Accumulation curve of bat species in six lagoons sampled at Parque Nacional da Restinga de Jurubatiba in 2003, Rio de Janeiro State, Southeastern Brazil: a) accumulated richness with lagoons; and b) accumulated richness with number of captures. 
Tabela 3. Dissimilaridade de Bray-Curtis e similaridade de Jaccard, em relação à fauna de morcegos, entre as seis lagoas estudadas no Parque Nacional da Restinga de Jurubatiba em 2003, Estado do Rio de Janeiro, sudeste do Brasil.

Table 3. Bray-Curtis dissimilarity and Jaccard similarity, in relation to bat fauna, among six lagoons sampled at Parque Nacional da Restinga de Jurubatiba in 2003, Rio de Janeiro State, Southeastern Brazil.

\begin{tabular}{lcccccc}
\hline & Cabiúnas & Comprida & Amarra-boi & Garças & Visgueiro & Piripiri \\
\hline Cabiúnas & - & 0,200 & 0,333 & 0,333 & 0,143 & 0,333 \\
Comprida & 0,818 & - & 0,200 & 0,333 & 0,143 & 0,444 \\
Amarra-boi & 0,787 & 0,800 & - & 0,500 & 0,333 & 0,333 \\
Garças & 0,708 & 0,561 & 0,673 & - & - & 0,844 \\
Visgueiro & 0,840 & 0,667 & 0,687 & 0,697 & 0,286 \\
Piripiri & 0,636 & 0,661 & 0,507 & 0,297 & 0,804 & - \\
\hline
\end{tabular}

Os valores na Tabela 3 abaixo e à esquerda referem-se ao coeficiente de dissimilaridade Bray-Curtis baseado na composição e abundância das espécies de morcegos nas lagoas amostradas do PNRJ. Quanto menor o coeficiente maior a similaridade da composição e da abundância de morcegos entre as lagoas. Os valores acima e à direita referem-se aos valores do índice de similaridade de Jaccard, baseado na presença/ausência das espécies nas lagoas amostradas do PNRJ. Quanto maior o índice, maior a similaridade da composição de espécies de morcegos entre as lagoas.

Values in the Table 3 below and in the left refer to the coefficient of Bray-Curtis dissimilarity based on the composition and abundance of bat species in the lagoons sampled in PNRJ. The smaller the coefficient the greater the similarity of the composition and abundance of bats between the lagoons. Values above and in the right refer to the Jaccard similarity index based on presence/absence of species in the lagoons sampled in PNRJ. The higher the index the greater the similarity of the composition of bat species among lagoons.

César Vinha (ES) com um esforço de captura de 40.300 h.m² e encontraram predominância de A. lituratus. Fogaça \& Reis (2008), com um esforço de captura de 48.960 h.m² , capturaram 129 indivíduos de 12 espécies no Parque Florestal do Rio da Onça (PFRO), no Paraná, e novamente a espécie predominante foi A. lituratus.

O número de espécies encontradas neste estudo foi menor que o observado por Luz et al. (2009), que com um esforço maior, mas obtendo eficiência menor $\left(0,006\right.$ capturas $\left./ \mathrm{h} . \mathrm{m}^{2}\right)$, registraram 17 espécies para a restinga de Praia das Neves. Dessas, nove espécies (G. soricina, C. perspicillata, Artibeus fimbriatus Gray, 1838, A. lituratus, Platyrrhinus lineatus (E. Geoffroy, 1810), P. recifinus (Thomas, 1901), U. magnirostrum, N. leporinus e M. nigricans) foram comuns a ambas as restingas (Luz et al. 2009).

Com base nestes trabalhos é possível afirmar que em ambientes de restinga é observada uma reduzida eficiência de captura, como observado por Oprea et al. (2009) e sugerido por Nogueira et al. (2010). Tal fato pode ser decorrente do ambiente mais aberto e vegetação mais baixa que pode permitir que os animais usem várias direções para seu deslocamento e não se concentrem em trilhas ou sobre riachos que se mostram mais produtivos para captura de morcegos em áreas florestadas (veja Kunz \& Kurta 1988). Outra hipótese é que em ambientes de restingas, as densidades de morcegos sejam menores.

Carollia perspicillata foi a espécie com o maior número de capturas (55 indivíduos) no PNRJ, diferente do observado por Fogaça \& Reis (2008), Luz et al. (2009) e Oprea et al. (2009), que encontraram A. lituratus como a espécie dominante em outras áreas de restinga, e Esbérard (2009), em ilha costeira. Porém, na Ilha Grande, C. perspicillata também foi a espécie dominante (Esbérard et al. 2006). Sturnira lilium (E. Geoffroy, 1810), espécie comum em parte dos inventários no Estado do Rio de Janeiro (e.g., Baptista \& Mello 2001, Esbérard et al. 2006, Modesto et al. 2008, Esbérard 2009), não foi capturada no PNRJ, e sua ocorrência também não foi registrada para a restinga de Praia das Neves (Luz et al. 2009). Talvez um esforço maior em ambas as restingas revele a presença de tal espécie, provavelmente em baixas densidades ou talvez esta espécie esteja presente somente em parte do ano. A presença de $S$. lilium já foi verificada em restingas do sul do Brasil (Fogaça \& Reis 2008, Carvalho et al. 2009).
Myotis nigricans (27 capturas) foi a segunda espécie mais comum encontrada no PNRJ, seguida por A. lituratus (25 capturas). Myotis nigricans foi capturado em redes dispostas em cima de corpos d'água. É possível que a grande quantidade de insetos presentes nestes ambientes maximize as taxas de captura da espécie (Esbérard \& Bergallo 2008, Lourenço et al. 2010), uma vez que ela é estritamente insetívora. Outro fator de importância para o aumento na captura dessa espécie é a proximidade com os centros urbanos, devido à diversidade de refúgios (naturais e artificiais) e à disponibilidade de insetos atraídos por luzes urbanas (Bredt et al. 1996, Silva et al. 1996). As quatro lagoas onde essa espécie foi capturada encontram-se muito próximas a construções antrópicas, ao contrário das lagoas Cabiúnas e Comprida, mais afastadas, com baixas capturas registradas.

Phyllostomidae foi a família mais abundante $(77,18 \%$ da riqueza) e diversificada no PNRJ, representada por todas as cinco subfamílias: Carolliinae, Stenodermatinae, Glossophaginae, Phyllostominae e Desmodontinae. A metodologia de coleta através de redes de neblina favorece a captura dessa família (e.g., Kunz \& Kurta 1988). Vespertilionidae foi a segunda família mais abundante $(18,79 \%)$, seguida de Noctilionidae $(4,03 \%)$. Locais bem amostrados no bioma da Mata Atlântica, em Mata Ombrófila Densa, resultam em um número de espécies de morcegos que varia de 20 a 40 (e.g., Esbérard 2003, Bergallo et al. 2003, Lourenço et al. 2010). Bergallo et al. (2003) sugerem um mínimo de 1000 capturas para amostrar com redes de neblina a maioria das espécies de filostomídeos de uma área. As áreas de restinga estudadas até o momento ainda não estão bem amostradas quanto à fauna de morcegos. Assim, espera-se que com o aumento do esforço amostral mais espécies sejam adicionadas às listas existentes para esses ambientes.

Grande parte das espécies capturadas no PNRJ pertence à guilda dos morcegos frugívoros. Alguns frutos comumente encontrados na dieta de morcegos, como espécies dos gêneros Ficus (Moraceae), Cecropia (Urticaceae) e Piper (Piperaceae), são encontrados na região (Pereira \& Araújo 2000). Já outros gêneros vegetais, muito comuns em ambientes de restinga, como Cereus, Opuntia e Pilocereus (Cactaceae), Andira e Inga (Leguminosae), possuem frutos carnosos e ainda podem ampliar a oferta de alimento para as espécies frugívoras e podem explicar a riqueza de frugívoros (Fábian et al. 2008).

A reduzida riqueza de Vespertilionidae e a ausência de Molossidae devem-se possivelmente à seletividade inerente ao método de captura. 
As espécies pertencentes a essas duas famílias têm hábitos insetívoros e possuem um sistema de ecolocalização mais apurado, sendo capazes de detectar as redes (Schnitzler \& Kalko 2001) ou voar a alturas maiores que as redes armadas. Porém espécies de Molossidae, como por exemplo, Molossus molossus (Pallas, 1766) podem ser capturadas em abundância sobre corpos de água (e.g., Lourenço et al. 2010). Como não foi empregada nenhuma outra metodologia, a não ser a captura com redes de neblina, apenas duas espécies primariamente insetívoras foram capturadas, $M$. nigricans e $L$. cinereus. O uso de outros métodos de amostragem, como busca ativa em abrigos e gravação de som por meio de detectores de morcegos, poderia adicionar novas espécies à lista de morcegos do PNRJ (Esbérard \& Bergallo 2008).

Glossophaga soricina é a única espécie na lista que apresenta hábito nectarívoro, e é a quarta espécie mais abundante $(\mathrm{N}=17)$. Esta espécie se alimenta também de insetos e frutos e é considerada a mais generalista dentre as espécies dessa guilda (Heithaus et al. 1975, Zortéa 2003). Espécies que se alimentam de néctar estão mais suscetíveis às mudanças sazonais devido à flutuação na disponibilidade do recurso (Zortéa 2003), sendo assim é possível que a realização de coletas na época chuvosa aumente a riqueza desta guilda.

Noctilio leporinus é uma espécie de hábito piscívoro e se alimenta também de insetos que voam sobre a superfície da água (Zortéa \& Aguiar 2001). Desta forma, redes armadas sobre ou próximo às lagoas têm maior chance de capturar essa espécie. No entanto, neste estudo $N$. leporinus foi capturada em apenas uma das seis lagoas.

Apesar do pequeno esforço de captura registrado neste estudo, foram capturadas cinco espécies pouco frequentes em inventários de morcegos (U. magnirostrum, L. cinereus, L. brasiliense, C. villosum e D. youngi). Tais espécies já haviam sido classificadas como localmente raras em outros estudos no Estado do Rio de Janeiro (e.g., Esbérard et al. 2006, Esbérard 2009, Luz et al. 2009).

Apesar da riqueza de espécies não ter diferido muito entre as lagoas (excetuando Visgueiro), a composição de espécies variou, sugerindo que amostragens em outras lagoas do PNRJ e no seu entorno possam aumentar a riqueza geral da restinga. Além disso, o fato da curva de acumulação de espécies não ter alcançado a assíntota também indica que com o aumento do esforço amostral mais espécies serão adicionadas à lista.

A diferença na composição de espécies observada entre as lagoas provavelmente se deve à grande heterogeneidade observada nos fatores abióticos das lagoas (Enrich-Prast et al. 2004) e à adaptação das espécies a esses fatores. As lagoas apresentam diferenças em relação à vegetação circundante (Pimentel et al. 2007), o que influencia diretamente na disponibilidade de insetos, e à comunidade de peixes (Caramaschi et al. 2004). Desta forma, a disponibilidade de alimentos para os morcegos varia entre elas e pode ser responsável por parte das diferenças encontradas. A lagoa Visgueiro, que apresentou a menor riqueza de espécies (apenas duas), é também a que apresenta a maior concentração salina e o maior $\mathrm{pH}$ encontrado no PNRJ. As espécies capturadas variaram muito entre as lagoas, o que mostra a importância desses ambientes para a manutenção da diversidade de morcegos, no entanto estudos mais aprofundados sobre as diferenças entre as lagoas e outros habitats da restinga se fazem necessários.

\section{Agradecimentos}

A Bat Conservation International e Secretaria de Educação de Quissamã pelo custeio e apoio. A Ana A. Barcelos e ao NUPEM/UFRJ pelo apoio e permissão de uso de instalações. Dr. Oscar Barbosa e Dra. Rosana Mazzoni pelas críticas a uma das versões deste manuscrito. A Daniel Raíces, Marco Aurélio Mello e Tássia Jordão-Nogueira pelo trabalho em campo. Trabalho desenvolvido sob licença especial para coleta do IBAMA-DF (processos 1755/89 e 4156/95-46). J.L. Luz,
R. Mangolin, H.G. Bergallo e C.E.L. Esbérard agradecem as bolsas de doutorado do $\mathrm{CNPq}$, de mestrado do CNPq, de Produtividade em Pesquisa do CNPq, Cientista do Nosso Estado da FAPERJ e Prociência/UERJ e Produtividade em Pesquisa do CNPq e Jovem Cientista do Nosso Estado da FAPERJ, respectivamente.

\section{Referências Bibliográficas}

ARAÚJO, D.S.D., SCARANO, F.R., SÁ, C.F.C., KURTA, B.C., ZALUAR, H.L.T., MONTEZUMA, R.C.M. \& OLIVEIRA, R.C. 1998. As comunidades vegetais do Parque Nacional da Restinga de Jurubatiba, Macaé, RJ. In Ecologia de restingas e lagoas costeiras (F.A. Esteves \& L.D. Lacerda, eds). UFRJ, Rio de Janeiro, p.39-62.

BAPTISTA, M. \& MELLO, M.A.R. 2001. Preliminary inventory of the bat species of the Poço das Antas Biological Reserve, RJ. Chiropt. Neotrop. 7(1-2):133-135.

BERGALLO, H.G., ESBÉRARD, C.E.L., MELLO, M.A.R., LINS, V., MANGOLIN, R., MELO, G.G.S. \& BAPTISTA, M. 2003. Bat species richness in Atlantic Forest: what is the minimum sampling effort? Biotropica 35(2):278-288.

BERGALLO, H.G.; MARTINS-HATANO, F.; RAICES, D.S.; RIBEIRO, T.T.L.; ALVES, A.G.; LUZ, J.L.; MANGOLIN, R. \& MELLO, M.A.R. 2004. Os mamíferos da Restinga de Jurubatiba. In Pesquisas de longa duração na Restinga de Jurubatiba - Ecologia, história natural e conservação (C.F.D. Rocha, F.A. Esteves \& F.R. Scarano, eds). RiMa, São Carlos, p.215-230.

BREDT, A., ARAÚJO, F.A.A., CAETANO-JÚNIOR, J., RODRIGUES, M.G.R., YOSHIZAWA, M., SILVA, M.M.S., HARMANI, N.M.S., MASSUNAGA, P.N.T., BÜRER, S.P., PORTO, V.A.R. \& UIEDA, W. 1996. Morcegos em áreas urbanas e rurais: manual de manejo e controle. Fundação Nacional de Saúde, Brasília.

CARAMASCHI, E.P., SANCHEZ-BOTERO, J.I., HOLLANDACARVALHO, P., BRANDÃO, C.A.S., SOARES, C.L., NOVAES, J.L. \& BARTOLETTE, R. 2004. Peixes das lagoas costeiras do norte fluminense: estudo de caso. In Ecologia de restingas e lagoas costeiras (F.A. Esteves \& L.D. Lacerda, eds). UFRJ, Rio de Janeiro, p.310-337.

CARVALHO, F., ZOCCHE, J.J. \& MENDONÇA, R.A. 2009. Morcegos (Mammalia, Chiroptera) em restinga no município de Jaguaruna, sul de Santa Catarina, Brasil. Biotemas 22(3):193-201.

CAUMETTE, P. 1992. Bacterial communities in coastal lagoons. An overview. Vie et Milieu 42(2):111-123.

CERQUEIRA, R. 2000. Ecologia funcional de mamíferos numa restinga do Estado do Rio de Janeiro. In Ecologia de Restingas e Lagoas Costeiras (F.A. Esteves \& L.D. Lacerda, eds). NUPEM/UFRJ, Macaé, p.189-214.

CERQUEIRA, R., FERNANDEZ, F.A.S. \& QUINTELA, M.F.S. 1990. Mamíferos da Restinga de Barra de Maricá, Rio de Janeiro. Museu de Zoologia da Universidade de São Paulo. Pap. Avulsos Zool. 37(9):141-157.

ENRICH-PRAST, A., BOZELLI, R.L., ESTEVES, F.A. \& MEIRELLES, F.P. 2004. Lagoas costeiras da Restinga de Jurubatiba: Descrição de suas variáveis limnológicas. In Pesquisas de longa duração na Restinga de Jurubatiba - Ecologia, história natural e conservação (C.F.D. Rocha, F.A. Esteves \& F.R. Scarano, eds). RiMa, São Carlos, p.245-253.

ESBÉRARD, C.E.L. 2003. Diversidade de morcegos em uma área de Mata Atlântica regenerada no sudeste do Brasil (Mammalia: Chiroptera). Rev. Bras. Zoocienc. 5(2):189-204.

ESBÉRARD, C.E.L. 2009. Capture sequence and relative abundance of bats during surveys. Zoologia 26(1):103-108.

ESBÉRARD, C.E.L. \& BERGALLO, H.G. 2008. Influência do esforço amostral na riqueza de espécies de morcegos no sudeste do Brasil. Rev. Bras. Zool. 25(1):67-73.

ESBÉRARD, C.E.L., JORDÃO-NOGUEIRA, T., LUZ, J.L., MELO, G.G.S., MANGOLIN, R., JUCÁ, N., RAÍCES, D.S.L., ENRICI, M.C. \& BERGALLO, H.G. 2006. Morcegos da Ilha Grande, Angra dos Reis, RJ, Sudeste do Brasil. Rev. Bras. Zoocienc. 8(2):147-153. 
ESTEVES, F.A. 1998. Lagoas costeiras: origem, funcionamento e possibilidades de manejo. In Ecologia das lagoas costeiras do Parque Nacional da Restinga de Jurubatiba e do Município de Macaé (RJ) (F.A. Esteves, ed.). NUPEM-UFRJ, Macaé, p.63-67.

FABIAN, M.E., RUI, A.M. \& WAECHTER, J.L. 2008. Plantas Utilizadas como alimento por Morcegos (Chiroptera, Phyllostomidae), no Brasil. In Ecologia de Morcegos (N.R. Reis, A.L. Peracchi \& G.A.S.D. Santos, eds). Technical Books Editora, Londrina, p.51-70.

FARJALLA, V.F., FARIA, B.M., ESTEVES, F.A. \& BOZELLI, R.L. 2001. Bacterial density and biomass. And relations with abiotic factors, in 14 coastal lagoons of Rio de Janeiro State. In Aquatic Microbial Ecology in Brazil. Series Oecologia Brasiliensis, vol. IX. PPGE-UFRJ, Rio de Janeiro, p.65-76.

FOGAÇA, F.N.O. \& REIS, N.R. 2008. Análise comparativa da quiropterofauna da restinga paranaense e adjacências. In Ecologia de Morcegos (N.R. Reis, A.L. Peracchi \& G.A.S.D. Santos, eds). Technical Books Editora, Londrina, p.87-95.

HAY, J.D., LACERDA, L.D. \& TAN, A.L. 1981. Soil cation increase in a tropical sand dune ecosystem due to a terrestrial bromeliad. Ecology 62(5):1392-1395.

HEITHAUS, S.R., FLEMING, T.H. \& OPLER, P.A. 1975. Foraging patterns and resource utilization in seven species of bats in a seasonal tropical forest. Ecology 56:841-854.

KALKO, E.K.V., HANDLEY, C.O. \& HANDLEY, D. 1996. Organization, diversity and long-term dynamics of a neotropical bat community. In Long-term studies of vertebrate communities (M.L. Cody \& J.A. Smallwood, eds). Academic Press, San Diego, p.503-553.

KUNZ, T.H. \& KURTA, A. 1988. Capture methods and holding devices. In Ecology and behavioral methods for the study of bats (Kunz, T.H., ed.). Smithsonian Institution Press, Washington, p.1-30.

LACERDA, L.D. \& ESTEVES, F.A. 2000. Restingas brasileiras: quinze anos de estudos. In Ecologia de restingas e lagoas costeiras (F.A. Esteves \& L.D. Lacerda, eds). UFRJ, Rio de Janeiro, p.III-VI.

LOURENÇO, E.C., COSTA, L.M., LUZ, J.L., DIAS, R.M. \& ESBÉRARD, C.E.L. 2010. Morcegos em manguezal - análise de uma assembléia e compilação de dados disponíveis no Brasil. In Mamíferos de restingas e manguezais do Brasil (L.M. Pessoa, W.C. Tavares \& S. Siciliano, eds). Sociedade Brasileira de Mastozoologia, Rio de Janeiro, p.173-187.

LUZ, J.L., COSTA, L.M., LOURENÇO, E.C., GOMES, L.A.C. \& ESBÉRARD, C.E.L. 2009. Bats from the Restinga of Praia das Neves, state of Espírito Santos, Southern Brazil. Check List 5(2):364-369.

MANGOLIN, R., MOTTA, A.G., ESBÉRARD, C.E.L. \& BERGALLO, H.G. 2007. Novos registros de Lophostoma brasiliensis Peters para o Sudeste do Brasil (Mammalia, Chiroptera, Phyllostomidae). Rev. Bras. Zoocienc. 9:225-228.

MODESTO, T.C., PESSOA, F.S., ENRICI, M.C., ATTIAS, N., JORDÃONOGUEIRA, T., COSTA, L.M., ALBUQUERUQE, H.G. \& BERGALLO, H.G. 2008. Mamíferos do Parque Estadual do Desengano, Rio de Janeiro, Brasil. Biota Neotrop. 8(4):153-159. http://www.biotaneotropica.org.br/ v8n4/pt/abstract?inventory+bn01408042008 (último acesso em 20/04/2011)

NOGUEIRA, M.R., MAZUREC, A.P. \& PERACCHI, A.L. 2010. Morcegos em restingas: lista anotada e dados adicionais para o norte fluminense, sudeste do Brasil (Mammalia, Chiroptera). In Mamíferos de restingas e manguezais do Brasil (L.M. Pessoa, W.C. Tavares \& S. Siciliano, eds). Sociedade Brasileira de Mastozoologia, Rio de Janeiro, p.75-93.
OPREA M., ESBÉRARD C.E.L., VIEIRA, T.B., PIMENTA, V.T., BRITO, D. \& DITCHFIELD A.D. 2009. Bat community species richness and composition in a restinga protected area in southeastern Brazil. Braz. J. Biol. 69:1073-1079.

PEREIRA, O.J. \& ARAÚJO, D.S.D. 2000. Análise florística das restingas dos Estados do Espírito Santo e Rio de Janeiro. In Ecologia de restingas e lagoas costeiras (F.A. Esteves \& L.D. Lacerda, eds). UFRJ, Rio de Janeiro, p.26-35.

PESSÔA, L.M., TAVARES, W.C. \& GONÇALVES, P.R. 2010. Mamíferos das restingas do macrocompartimento litorâneo da bacia de Campos, Rio de Janeiro. In Mamíferos de restingas e manguezais do Brasil (L.M. Pessoa, W.C. Tavares \& S. Siciliano, eds). Sociedade Brasileira de Mastozoologia, Rio de Janeiro, p.95-125.

PIMENTEL, M.C.P., BARROS, M.J., CIRNE, P., MATTOS, E.A., OLIVEIRA, R.C., PEREIRA, M.C.A., SCARANO, F.R., ZALUAR, H.L.T. \& ARAUJO, D.S.D. 2007. Spatial variation in the structure and floristic composition of "restinga" vegetation in southeastern Brazil. Revista Brasil. Bot. 30(3):543-551.

ROCHA, C.F.D. 2000. Biogeografia de répteis de restinga: distribuição, ocorrência e endemismos. In: Ecologia de restingas e lagoas costeiras (F.A. Esteves \& L.D. Lacerda, eds). UFRJ, Rio de Janeiro, p.99-116.

ROCHA, C.F.D, BERGALLO, H.G., ALVES, M.A.S. \& VAN SLUYS, M. 2004. A Restinga de Jurubatiba e a conservação dos ambientes de restinga do Estado do Rio de Janeiro. In Pesquisas de longa duração na Restinga de Jurubatiba - Ecologia, história natural e conservação (C.F.D. Rocha, F.A. Esteves \& F.R. Scarano, eds). RiMa, São Carlos, p.341-352.

SARAÇA, C.E., RAHY, I.S., SANTOS, M.A., COSTA, M.B. \& PERES, W.R 2007. A propósito de uma nova regionalização para o Estado do Rio de Janeiro. Rev. Econ. Flum. 6:18-27.

SCHNITZLER, H.U. \& KALKO, E.K.V. 2001. Echolocation by insect-eating bats. BioScience 51:557-569.

SILVA, M.M.S., HARMANI, N.M.S., GONÇALVES, E.F.B. \& UIEDA, W. 1996. Bats from the metropolitan region of São Paulo, southeastern Brazil. Chiropt. Neotrop. 2(1):39-41.

SOFIATTI, A. 1998. Aspectos históricos das lagoas do Norte Fluminense do Estado do Rio de Janeiro. In Ecologia das lagoas costeiras do Parque Nacional da Restinga de Jurubatiba no Municipio de Macaé (RJ) (F.A Esteves, ed.) UFRJ, Rio de Janeiro, p.3-35.

STRAUBE, F.C. \& BIANCONI, G.V. 2002. Sobre a grandeza e a unidade utilizada para estimar esforço de captura com utilização de redes-deneblina. Chiropt. Neotrop. 8(1-2):150-152.

TANIZAKI-FONSECA, K. \& MOULTON, T.P. 2000. A fragmentação da Mata Atlântica no Estado do Rio de Janeiro e a perda de biodiversidade. In A fauna ameaçada de extinção do Estado do Rio de Janeiro (H.G. Bergallo, C.F.D. Rocha, M.A.S. Alves \& M.V. Sluys, eds). EdUERJ, Rio de Janeiro, p.23-35.

ZORTÉA, M. 2003. Reproductive patterns and feeding habits of three nectarivorous bats (Phyllostomidae: Glossophaginae) from the Brazilian Cerrado, Braz. J. Biol., 63(1):159-168.

ZORTÉA, M. \& AGUIAR, L. 2001. Foraging behavior of the fishing bat Noctilio leporinus (Noctilionidae). Chiropt. Neotrop. 7(1-2):140-142. 
Luz, J.L. et al.

Apêndice 1. Espécimes testemunhos depositados na Coleção de Referência do Laboratório de Diversidade de Morcegos (LDM), Instituto de Biologia, Universidade

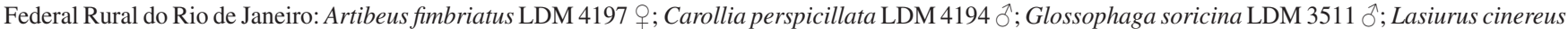

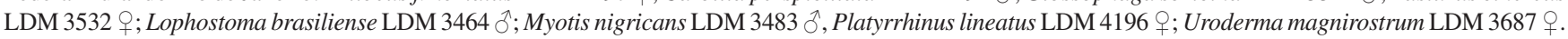

